\title{
Assessed quality of service and voice and data integration: A case study
}

\author{
Aurelio La Corte *, Sabrina Sicari \\ Department of Computer Science and Telecommunications Engineering, University of Catania, Viale Andrea Doria 6, I 95100 Catania, Italy
}

Received 8 August 2004; received in revised form 14 December 2005; accepted 18 December 2005

Available online 23 January 2006

\begin{abstract}
Quality of service (QoS) can be defined in many ways and can include various aspects and performance metrics. Assessed QoS reflects customer experiences about service and expectations in terms of costs, support of innovative features and return of investments. The adoption of a solution where voice and data are integrated into an IP-based network infrastructure leads users to a new degree of satisfaction having a bad impact on users' opinion in terms of Assessed QoS. In this paper, we present a practical case study of telecom convergence implemented into the university campus sites scattered throughout the metropolitan area of Catania. The QoS factors which have been considered in order to decide the adoption of a technical solution based on voice and data integration are also pointed out. (c) 2006 Elsevier B.V. All rights reserved.
\end{abstract}

Keywords: QoS; VoIP; Telecom convergence; Internet telephony

\section{Introduction}

In the last years information and communication technologies (ICTs) have had a remarkable development, resulting from the larger use of digital technologies related to Internet. This network becomes the core of globalization: it represents the communication system enabling to control nearly all the information types and to push a full diffusion of ICT technologies. The further development of this process, contributing to improve the efficiency and the effectiveness of the communication system, could be obtained by integrating all the media in a single system. In other words, the idea is to develop the communication system by means of a convergent process, in order to offer a better communication experience, better communication and resources management. Actually, in agreement with [16] we define a convergent process as multidisciplinary, inhomogeneous integration with the aim to reach an added value.The VoIP convergence started on the application level and resulted in a heterogenous integration of the technologies.

\footnotetext{
${ }^{*}$ Corresponding author. Tel.: +39 0957382356; fax: +390957382397.

E-mail addresses: aurelio.lacorte@unict.it (A.L. Corte), sabrina. sicari@unict.it (S. Sicari).
}

Various telecommunication market fields considerably evolve in this direction. For example, in Europe there is a strong impulse to experimentation and to the spread of digital audio and video broadcasting supported by interactive services. Moreover, all device vendors of networking and telephone systems, and generally of business communication systems, develop technological solutions that allow the convergence of real-time voice over data networks based on Internet Protocol (IP).

The idea to integrate voice and data networks is not new. The development of narrow-band and broadband Integrated Service Digital Networks (ISDN) results from such idea. At the same time, the structured cabling system allows the simultaneous development of voice and data transmission.

Telecom operators transmit voice and data services in an integrated way for a long time in their network, and some Internet service providers supply simple voice services to their own subscribers. Recently, instead, the convergence of voice, and data services also involves business customers, to whom it offers advanced and sophisticated services, based on computer and telephone integration. Today, an emerging generation of equipment, based on the network technology convergence, is deployed to carry voice, data, and video traffic. 
The implementation of the convergence of real-time voice services over data networks based on Internet Protocol involves several technical aspects [1]. Although voice is digitized and transmitted through a packet switched network based on Internet Protocol, there are two ways, each one very different from the other, to implement a communication system based on the transmission of Voice over Internet Protocol (VoIP). The first way, shown in Fig. 1, refers to the case in which PBXes of a company are connected to a VoIP gateway and a Public Switched Telephone Network (PSTN). In this case, the VoIP gateway allows on-net telephone calls to be completed through the IP network. The off-net calls are completed through the PSTN, and therefore only the interconnection of PBX involves the transmission of voice over an IP network.

The second way to implement a VoIP system, shown in Fig. 2, refers to the case in which the classical PBX is not used and the telephones are IP devices, called "IP phones". Calls between two IP phones of the same site are sustained by Local Area Network (LAN). Calls between different sites can be made over the wide area IP network, while calls towards users of PSTN are allowed by a VoIP gateway

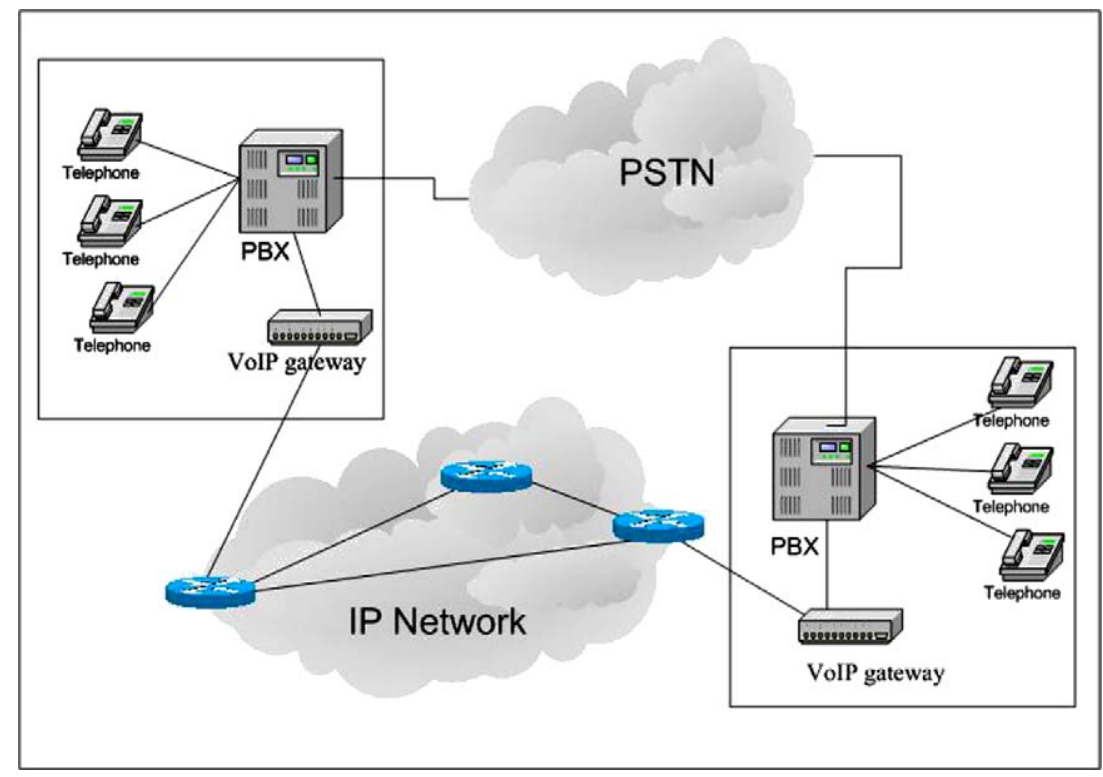

Fig. 1. Classical PBX's are interconnected by an IP network.

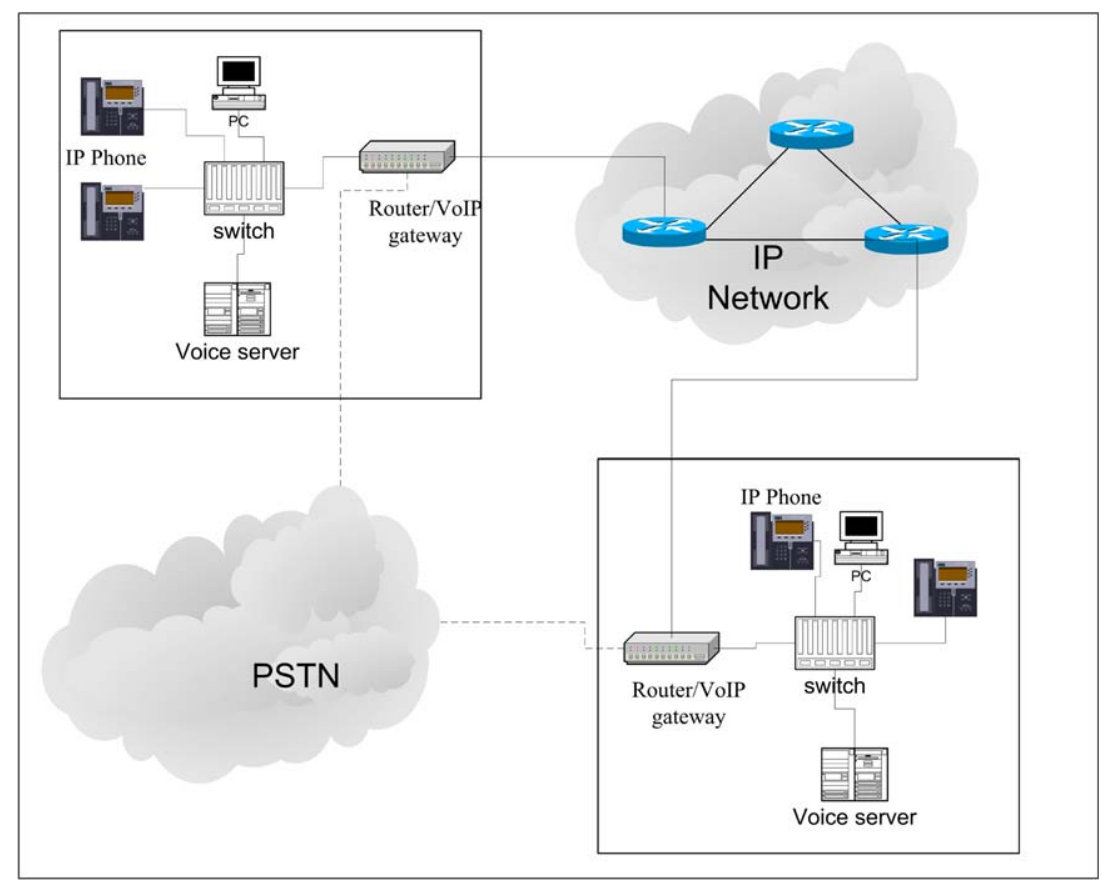

Fig. 2. A scheme of VoIP system. 
interconnected to PSTN. Telephone services are sustained by a voice server.

In this paper, we refer to a VoIP system as a communication system where voice and data are wholly integrated in a convergent network, in which telephones are IP devices (IP phones). Analogical modem and fax machines are interconnected by means of appropriate terminal adapters, which are also interconnected to the LAN as an IP device, and the telephone services are sustained by one or more servers: anyway, in our opinion, a VoIP system is a synonym of IP phone system.

Today, there are several solutions in the market to implement a VoIP system and several different service architectures. It is still unclear which VoIP architecture will meet user needs in the best way [2], which services will be the most successful, where these services have to be located and how they must be distributed in the network. In any case, from the technological point of view, VoIP is a radical technological improvement, for both users and TLC operators. Presently, the basic applications of a VoIP system are based on advanced codecs, packet switching, dynamic bandwidth allocation and sophisticated techniques for dynamic routing. The network is used in such an efficient way that it permits to by-pass the rating systems designed for traditional voice networks [3].

The reasons for implementing a convergent solution rather than a traditional one are related to the advanced services that are available to customers and to the cost of these services. Both of them affect the user quality perception of VoIP system. For telecom operators, companies and network administrators, VoIP systems represent new business opportunities allowing a reduction in management and maintenance costs of the communication system and lead to an increase in the value of network infrastructures. Such aspects are extremely interesting in the current ICT market, characterized by a considerable resizing of investments by companies and public administration.

In literature there are many works about VoIP system, somebody speak about speech quality others point out price, but our goal is different.We analyze advantages and disadvantages from a technical and economical point of view and customer judgement of the system. Actually, different kinds of customer are asked to express a judgement about service value.We want to offer a full judgement about this new technology; in other words, we evaluate assessed quality of service. Actually, the features of the VoIP system and the impact of this system on the implementation and management costs requires the revision of the QoS concept, as a function of the intrinsic characteristics of the system. Actually, QoS is not only related to such factors as delay, throughput, jitter and so on, but also to factors as:

- perception and expectation of services by the users;

- availability of innovative features;

- return of investment (RoI) that represents the profit or loss resulting from an investment transaction, usually expressed as an annual percentage return;
- user satisfaction degree of communication system and services.

The QoS, therefore, is related to the subscriber perception and to the network services and performance available when users start to use a new technology.

The costs for implementing a VoIP system are often comparable or superior to the cost of a traditional telephone system. However, a VoIP system is more flexible and supports new services; therefore, it allows TLC operators to use new billing strategies and, at the same time, it permits companies to plan, develop or use new sophisticated services based on ICT applications.

To estimate the investment costs for implementing a VoIP system, it is essential to consider the life span of VoIP systems today, being usually lower than the traditional telephone systems. This could seem to be a drawback, but, a big VoIP potentiality is represented by the possibility to exploit market dynamism and various technical solutions.

In this paper we want to present, also by means of a real case study, the main motivations that lead to the telephone system implementation based on a convergent solution with strict integration of voice service over an IP network, rather than of one implemented on the classic PBX. For it regards the economic aspects, the attention is focused on the return of investment, on the break-even-point (that represent the amount of money at which a system solution, in our case a VoIP solution, produces neither a gain nor a loss, in other word benefits are equal to the costs). This analysis is finalized to evaluate Assessed QoS that reflects user experiences of using a service, the expectation in terms of costs, innovative feature support and return on investment.

The paper is organized as follow: in Section 2 of the paper a synthetic overview of Italy ICT market in the last year is offered. As result of this panoramic the main actors who are interested to use of convergent solutions for voice and data services are carried to the light. In Section 3, the main pro and cons of a VoIP system are illustrated. In Section 4, the elements that have to be considered in economic valuation of VoIP system are also pointed out. In Section 5, an architectural model to represent QoS is introduced. Such model highlights the aspects connected to the new services and the economic advantages that derive from the utilization of VoIP systems. In Section 6, a case study is presented: this describes the VoIP system realized by the University of Catania within campuses that are distributed around metropolitan and regional territory. The paper describes the way by which has been reached the decision to develop University telephone system towards a VoIP convergent system. Some indications of customer VoIP QoS perception are given. In Section 7, we discuss related work. Finally, in Section 8, we draw some conclusions and sketch future work. 


\section{An outlook on ICT market}

The recent economic trend, at world and European level,causes a notable reduction in the ICT market. In Italy, the hardware market is heavily reduced, especially in 2002. Software and service fields, on the contrary, are not affected in the same way.

This derives also from the fact that in the previous years companies and public administrations greatly invested in information technology. Such investments were aimed at homogenizing company information systems, so as at facing the new technological paradigms and businesses relying more and more on the computer science technologies as the qualifying factors of business changes. During 2003 companies and public administrations planned investments on hardware and software resources following tough criteria and with more awareness because of an uncertain economic situation.

The telecommunication market also suffers from reductions in the last years, even if in different ways and in various fields. The incumbent operator finds favorable conditions in the market conditions to increase his efficiency and to protect his own market quotas. Instead, mobile phone operators are sustained by the growth of services in traditional market segments, based on voice, and short messages. All operators try to build models based on added value services to boost the request.

Therefore, the market appears to be less receptive in welcoming the opportunities offered by the technological evolutions of the wide band on fixed network and mobile network. It is not possible to take full advantage of the opportunities offered by Internet to improve the production and social wealth. This is mainly caused by the fact that, still today, nearly $50 \%$ of the Italian adult population does not utilize personal computer.

In a market that was strongly reorganized during the last years, attention is paid to new opportunities linked to the convergence of network technologies. This convergence means a synergic integration of sectors, markets, and products of various origin and sources. It concerns, for example, the convergence of voice/data/video into a single IP-based network architecture for all types of information and media. However, such perspectives have not to be evaluated in the short and medium term. Today video broadcast or video on-demand services on an IP-based network cannot be diffused to various areas of the national territory since in different areas of the country the performance of the access network does not allow customers to use such services. Furthermore, this kind of services is little spread in the companies. However, VoIP service continues to be largely welcomed. This technology allows us to consider voice network as strategic as data network [4].

During the last years, in the Italian ICT market, public administration played a key role. Various reforms were launched:
- decentralization, efficiency, and effectiveness of the public function;

- quickness and swiftness of procedures;

- act rightness;

- administration procedure simplification.

Today, public administration appears to be as one of the main actors in the market of "convergent networks", carrying out the front-office function for several integrated services to the citizens.

In order to reach modernization, information technologies, mainly those tied to Internet, play a major role. In particular, the initiative of e-government has a key role also because this has clearly a remarkable influence inside the investment strategy in information technology on behalf of public administration.

\section{Reason of convergent solution}

Today, the market offers various products and various technological solutions in order to realize VoIP systems and, in general, convergent systems. A common element to all these solutions is the interpretation of a company telephone system. Nowadays, a VoIP system is usually seen as a distributed system allowing the following advantages:

- scalability and flexibility;

- availability;

- easy management and configuration of users and network devices;

- increase in productivity.

These advantages result from the use of the Internet Protocol and from the possibility to implement, through the telephone system, new services. Moreover, some advantages indirectly derive from the need that the VoIP system implementation requires a careful development planning of the communication network.

The characteristics of scalability and flexibility of the VoIP systems are caused by the distributed nature of the architecture which they are based on, which therefore makes them strongly scalable in terms of amount of customer interfaces. The activation of a new customer does not interfere with the normal running of the network. Moreover, it is possible to configure new customers by using directory repository logic in the same way as they are added to one directory. In this way scalability is guaranteed. At the same time, the IP use assures flexibility in terms of increase of its configuration and system internal communication. The system itself is able to dynamically adapt to the migration of services and applications, the resource reallocation, the customer internal mobility and the virtual subnet reconfiguration, without interfering with the ordinary network running.

Before audio or video media can flow between two computers, various protocols must be employed to find the remote device and to negotiate the means by which media 
will flow between the two devices. The protocols that are central to this process are referred to as call-signaling protocols, the most popular of which are H.323 and Session Initiation Protocol (SIP). Both H.323 and SIP can be referred to as "intelligent endpoint protocols". What this means is that all of the intelligence required to locate the remote endpoint and to establish media streams between the local and remote device is an integral part of the protocol. There is another class of protocols which is complementary to H.323 and SIP referred to as "device control protocols". Those protocols are H.248 and MGCP. To understand the purpose of H.248 and MGCP, it is important to first understand the function of a gateway. A gateway is a device that offers an IP interface on one side and some sort of legacy telephone interface on the other side. The legacy telephone interface may be complex, such as an interface to a legacy PSTN switch, or may be a simple interface that allows one to connect one or a few traditional telephones. Depending on the size and purpose of the gateway, it may allow IP-originated calls to terminate to the PSTN (and vice-versa) or may simply provide a means for a person to connect a telephone to the Internet. Originally, gateways were viewed as monolithic devices that had call control (H.323/SIP) and hardware required to control the PSTN interface. In 1998, the idea of splitting the gateway into two logical parts was proposed: one part, which contains the call control logic, is called the media gateway controller (MGC) or call agent (CA), and the other part, which interfaces with the PSTN, is called the media gateway (MG). With this functional split, a new interface existed (going between the MGC and MG), driving the necessity to define MGCP and H.248. Telephony call control is a critical part, actually for correctly handling any speech input and output, applications must interact with the phone system for basic actions such as answering, making, transferring, and disconnecting calls. In addition, some applications require more complex extended call control actions such as setting up three-way consultation calls in order to conduct supervised transfers or conference calls.

Thanks to media gateway controller, a VoIP system is able to guarantee a reliability level comparable to that of classical PBX systems, with a level of flexibility being definitely advanced compared to the traditional PBXes.

Reliability derives from the fact that a good plan of VoIP system takes into account advanced solutions for IP-based networks, in order to resolve breakdowns on nodes and connections. The Internet Protocol allows the use of different paths for reaching every data destination, and therefore, also in case of entire network section failure, the automatic routing strategy is able to find an alternative path.

However, it is important to observe that the classic PBX reliability should be considered itself an intrinsic characteristic. At present, PBX is based on a wide solid technology over time and on a faithful architecture. Instead, in the case of the VoIP systems, the reliability is achieved taking advantage of a high level of redundancy on the elements composing the system. Obviously, all of this increases costs.

One of the main reasons for the VoIP system success is the simplicity of management and administration by customers. The Web interface is exploited in different ways: network device management, configuration of personal/ general parameters, and interaction with telephone services. The customers can configure some parameters by means of a browser without a system administrator help. This is definitely different from the traditional systems, that, although having many functionalities which are hardly ever practised daily, nearly always need the participation of vendor skilled technicians in order to carry out the personalization.

The use of only one technological infrastructure for both data transmission and distribution of telephone services contributes to a significant cost reduction for network management and maintenance. All configuration activities, caused by customer moving, system modification, change of customer profile, and related qualifications, changes of the correspondence between customer and its own telephone number, particular settings of additional services (voice-mail, applications, and call redirection), are eliminated by binding together a structured cabling system and user mobility services with the VoIP solution. Besides, it achieves a cost reduction of management and maintenance of a VoIP system compared to a traditional PBX.

The benefit that derives from the cost reduction for adding, moving, and modifying customers is as important as the benefit achieved by the easiness to insert new applications into a VoIP system. New applications contribute to increase customer productivity. Presently, VoIP systems contribute to optimize various parameters, both organizational, and technological ones. In particular, the flexible organization allows fast movements of equipment, new aggregations of work groups, decentralization of activity and responsibility and also the implementation of new added value services.

Among the advantages expected from the adoption of a VoIP system, it is very important to remind the controlling of on-net calls. The toll bypass is still a strong factor in the choice of a convergent system. This contributes to a cost reduction of on-nets calls than PSTN. Actually, for multi-location businesses, inter-office calling typically represents $25-40 \%$ of a company's total long distance bill. To bypass long distance charges, each office installs and configures a MultiVoIP on their network to place calls, send faxes, or make modem connections to the other offices on the VoIP network; this technique is called toll bypass. The most substantial savings are often realized in overseas communication. However, the reduction of traditional telephone tariffs over time determines a loss of the importance of such factors when choosing a convergent system. Finally, by combining voice and data there is a significant reduction of staffing requirements.

There are also drawbacks in the choice of a convergent solution, which must be taken into account [4]. The main 
obstacle seems to be the lack of budget. The transformation of a classic PBX into a VoIP system involves an investment that often turns out to be a remarkable one and in the short run, it is not justified in terms of resulting advantages.

The second drawback is the insufficient interoperability among vendor equipment. Although being a problem also in the case of traditional PBXs, the customers hopes that VoIP systems have the same level of interoperability that the systems for data transmission have today.

Finally, a third obstacle turns out to be security. In a convergent system attacks on data network could be also translated into improper functioning of telephone services. The choice of a convergent system demands a careful analysis of security problems in the network, and therefore, sometimes, also a data network reorganization.

\section{Total cost of network ownership and break-even-point analysis}

The advantages and disadvantages deriving from the use of a VoIP system with respect to a system based on traditional PBX have impact on the costs that must be supported in order to realize a system. At present, the decision to adopt a convergent solution derives from a careful cost analysis, both direct and indirect, and from the economic advantages measured in a smaller money expenditure for the company that invests. These costs must be compared to the benefits deriving in terms of services. Therefore, the investment in a new technology is mainly motivated by two factors:

- early and annual cost reduction of infrastructure;

- productive capacity or quality improvement of the internal company processes.

The economic tool that allows to evidence the total cost that a company must support, in order to replace its own technological park, is the TCO (Total Cost of network Ownership). This parameter is given from the sum of the following costs:

- system planning;

- net infrastructure realization;

- hardware and software purchase necessary to implement the system;

- device installation;

- configuration and administration of system and services;

- customer technical support;

- infrastructure and device maintenance;

- security procedure adaptation of data transmission system in order to protect the telephone system.

Among the cost items, indicated above, there are those linked to customer activities of move, add, and change. In order to point out the economic advantages, i.e., money saving over time, an analysis of break-even-point must be carried out. This analysis indicates the time necessary to obtain a return of the investments, and particularly the minor costs annually supported on the new network infrastructure compared to the traditional ones.

In order to determine the break-even-point it is necessary to analyze the following costs:

- material supply;

- labour employed to realize the system;

- convergent system maintenance;

- customer training for the new system;

- telephone traffic.

It is important to point out that by using a convergent solution there is a "single" network able to manage both voice and data. Consequently, in the cost analysis, it is necessary to take into right account also the costs, or the savings that are indirectly obtained on data transmission system.

In the Section 6 a case study will be presented and will be given some numerical values on parameters indicated above.

\section{Quality of service}

QoS can be defined in many ways and can include various aspects and different sets of service requirements, such as performance, availability, reliability, and security. The parameters describing QoS can be defined in both deterministic and stochastic ways or by means of average values at suitable time intervals [5].

QoS and its parameters can take on a meaning, depending on how to evaluate it. Customers, service providers, and telecommunication engineers view QoS in a different way, using performance metrics for QoS evaluation which can be different one from the other. At the same time, the customer opinion about QoS of a given service and his satisfaction level depend on his familiarity with the service and the computer science technologies.

In [6] a general model is presented. In this model the notions of Intrinsic, Perceived, and Assessed QoS are shown. Intrinsic QoS is strictly determined by transport network design and the provision of network access, termination, and connection [6,7]. The approach used to define QoS is similar to that one used by IETF for the definition of QoS [8-10] and for the approach used by ITU and ETSI to define the concept of Network Performance [11-14].

Perceived QoS takes into account how the service is perceived by the customer. It is a subjective quality evaluation and, therefore, can be defined through both technical and objective parameters, linked to the parameters representing the Intrinsic QoS and the user expectations of a specific service. Apart from the technical aspects, linked to the notion of Network Performance, the approach followed by ITU and ETSI to define QoS is essentially based on its perception by the user $[13,11]$. However, the IETF approach is completely different and, as already mentioned, it deals with the QoS problem as an intrinsic QoS problem and it pays no attention at all to the perceived QoS. 
The Assessed QoS represents a vision of perceived QoS from a higher level than perceived QoS and it depends, apart from the perceived QOS, on various factors such as service, price and client satisfaction based on customer care [6]. Neither ITU nor ETSI norvIETF deal with the Assessed QoS [7].

An overview of commonly used terminology related to the quality of service in IP networks and a comparison among the approaches used by IETF, ITU, and ETSI for defining QoS are shown in [7].

In this paper, taking what is reported in [7], we introduce a model allowing us to highlight the QoS aspects also in a communication environment where the convergence of the circuit switched and packet switched networks in IP-based infrastructures is implemented. Hence, the term QoS has several meanings, ranging from a user service perception to a set of connection parameters being necessary to achieve a particular service quality.

Let us consider the layered structure of QoS representation, as shown in Fig. 3.

The two lowest layers are the Basic Level and the Multimedia Level. The Basic Level corresponds to the layer of Intrinsic QoS in the general model proposed in [7], and identifies the QoS parameters that must be considered in any type of connection. These depend on the technical aspects and are determined by the type of projected transport network, from connection to termination. QoS is expressed in terms of user data transfer bit rate that is available to the service or target throughput that may be achieved, delay and delay variation (jitter) experienced by user information units while passing through the network and user information unit loss rate [7].
The Multimedia Level is the second layer in the proposed model. This layer was introduced taking into account the requirements of multimedia services and essentially refers to media synchronization. At present, a multimedia stream is characterized by multiple monomedia streams related to each other by means of time, spatial or logical relationships that can be altered when the information unit crosses a network and that must be preserved [15]. At this level, the QoS parameters are basically linked to the skew occurring in the multimedia stream, that is the difference between the instantaneous delays of information units belonging to two different monomedia streams composing the multimedia stream, in other words skew refers to the timing difference which occurs when information units which are traveling over different pathes reach their destinations at different times. The different arrival times of the information unit may present a problem when simultaneous arrival with no delay is required, as in multimedia application. As shown by the human perception measurements of the above parameters, monomedia streams may appear to be "in synch" if jitter and skew are limited to appropriate values [17], the QoS parameters can be expressed as restrictions of skew statistic values.

The third level proposed is the Wireless Level. This level was introduced to measure the user mobility, or, more specifically, to take into account the fact that when a mobile terminal passes from one cell to another the information unit rerouting may induce a sudden variation in the endto-end delays, and, depending on the technique used for the handover, a loss or duplication of the information units making up each monomedia stream. At this level, we assume that the mobile terminal (MT) carries out the hand-

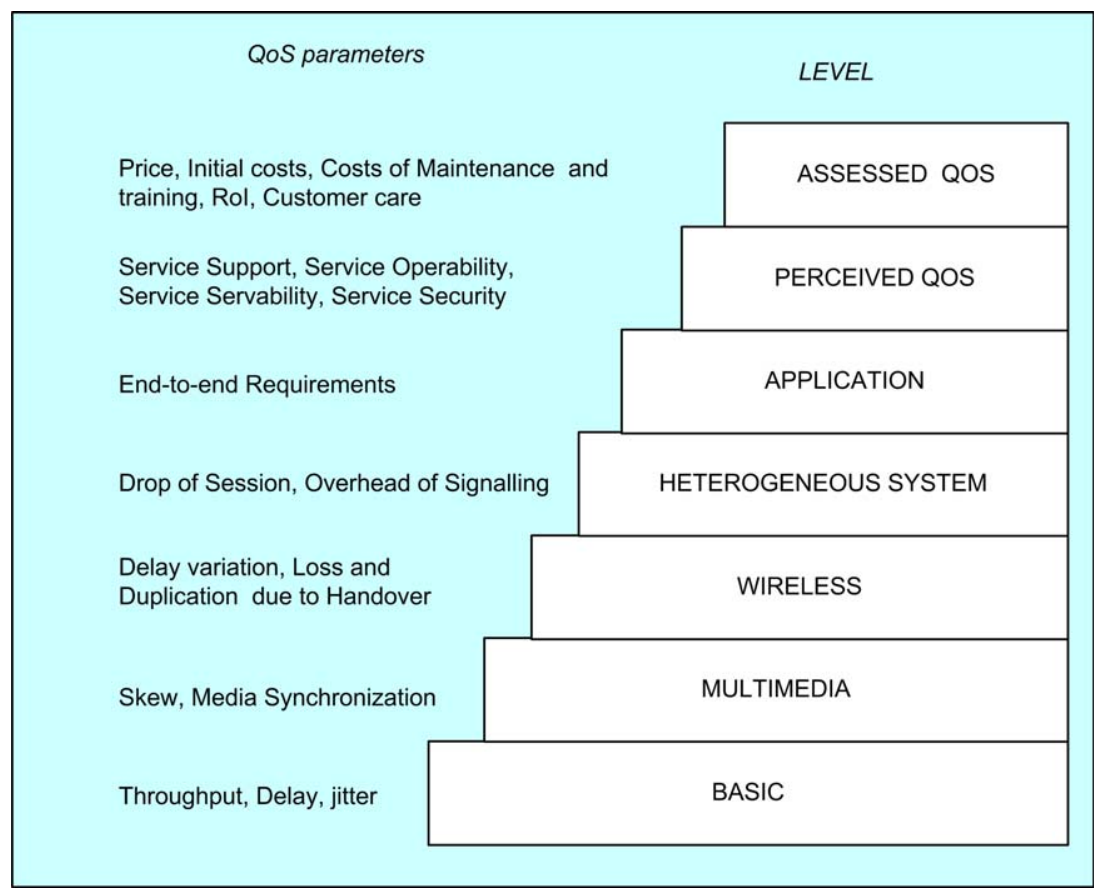

Fig. 3. General model of QoS. 
over through the homogeneous systems and, in this way, it does not carry out a network protocol change during the passage from one cell to another. Therefore, the connection nominal rate does not change. The QoS parameters can be expressed in terms of variation of delay average value during the passage from one cell to another and in terms of the number of information units that are lost or duplicated during the handover. Also in this case, the QoS parameters can be expressed as restrictions of the statistics values [15].

The fourth level of the proposed architecture is the Heterogeneous System Level. This takes into account the fact that the handover can be carried out by heterogeneous systems. In this case, the mobile terminal has to carry out a change in network access protocol, and a mechanism guaranteeing session continuity must be set up. QoS, at this level, can be represented by two parameters: the probability that the session will not be maintained, and overhead in the transmission due to the signaling necessary to maintain the session. The probability of a drop in the session depends on the type of network access protocol and other factors such as the MT speed, nominal rate variation and so on.

At the next level of the QoS model architecture we find the Application Level. This level takes into account the service specific characteristics. The QoS parameters are linked to the application and are expressed in terms of type of end-to-end requirements. For example, in the case of Web Browsing, the QoS parameter is the response time, while in the case of VoIP services, it is the end-to-end delay.

At the next level of the model shown in Fig. 3 we find the Perceived QoS Level. This takes into account how the user perceives the QoS in his/her experience using the service and is influence by the customer expectations as opposed to observed service performance [7]. These expectations reflect how the service is supported, and the performance in terms of operability, service ability, and service security. It can be compared to its homonyms in ITU and ETSI models. The Perceived QoS derives from subjective aspects. It also depends on the customer familiarity with the service use, or on the customer attitude towards new technologies.

At the highest level of the model the Assessed QoS Level is located. The Assessed QoS is a fundamental parameter, because the customer decision to continue using or not using the service depends on it $[7,6]$. This depends on many factors: the quality perceived by the user, system and service availability and reliability, pricing, initial costs, return of investments (RoI), billing accuracy, customer care responses, and benefits depending on the service use in economic terms as well as in productive ones. Therefore, the Assessed QoS also depends on how the expectations related to the return of the investments or advantages resulting from the use of a given technology are met. For example, if the company chooses a convergent solution with a management staff reduction goal and if the objective is achieved, the customer opinion, in terms of Assessed QoS, will be negative, independently of the effective quality of the system. And still, if the company buys extremely functional IP telephones, configurable and personalized via Web-based interface, but the user has insufficient familiarity with ICT technologies, the customer judgement on the VoIP system will be once again a negative one, even if the system administrator considers it very easy to manage.

Therefore, the Assessed QoS does not really express a QoS metrics but rather the user full judgement, taking into account the perception of a system or of a service and the customer expectations. The Assessed QoS can be low, though the QOS performance metrics of the lowest levels shown in Fig. 3 are high, if the user considers the relationship cost/performance very bad. At the same time, the Assessed QOS can be high or low for the same service, according to the service usefulness for the user.

Therefore, the success of a convergent system is strictly linked to the Assessed QoS, expressing the full judgement on quality. The lowest levels of the architecture shown in Fig. 3 contribute to determine the value of Assessed QoS; but the value that assumes depends also on an right planning of the whole system, orienting the services provided by the system to the receiver users.

\section{Case study of University of Catania}

Presently, the University of Catania plays an important role on the territory, also due to the large number of people it includes: 65,000 people, including teachers, students, and administrative staff. Its expansion results from an investment policy enhancing the teaching power and quality and attracting, as a consequence, new registrations. This has pushed the Athenaeum to decentralize the University campus sites in other Sicilian cities, thus boosting the access to study of other province young people.

The University of Catania has various departments in the urban area of Catania and various centres in Eastern Sicily, being nearly $10-120 \mathrm{~km}$ away from the headquarters. Catania University site is divided into departments located in several urban areas (see Fig. 4).

Recently, in order to include the largest number of detachments in a single network, a broadband fibre optic network was created. The network infrastructure is based on $23 \mathrm{~km}$ of cables of 100 and 20 optical fibres. It extends on a territory of nearly $16 \mathrm{~km}^{2}$ in the urban area of Catania.

The network architecture that interconnects various university departments is shown in Fig. 5. The transport network is based on 10 Add Drop Multiplexer SDH STM-16 (2.5 Gbit/s). Access network is based on Ethernet protocol and ATM (155 Mbit/s) backbones for the sites in the urban area, while it relies on xDSL technology for the regional sites. The servers for the different services (e-learning, VoIP, and so on) are located in the various sites of the transport network.

Until 2002, in all university sites, the traditional PBXes were installed. Typically, each university department has 


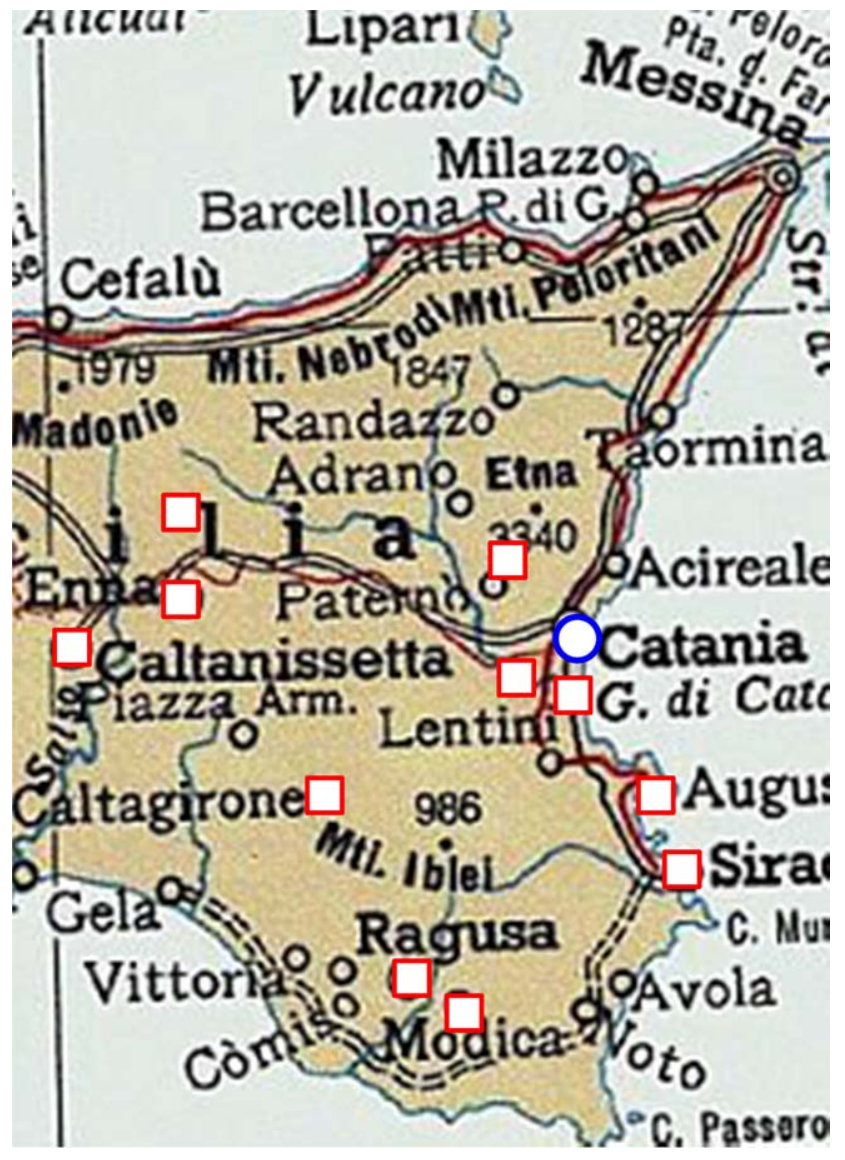

Fig. 4. Catania University sites in Eastern Sicily.

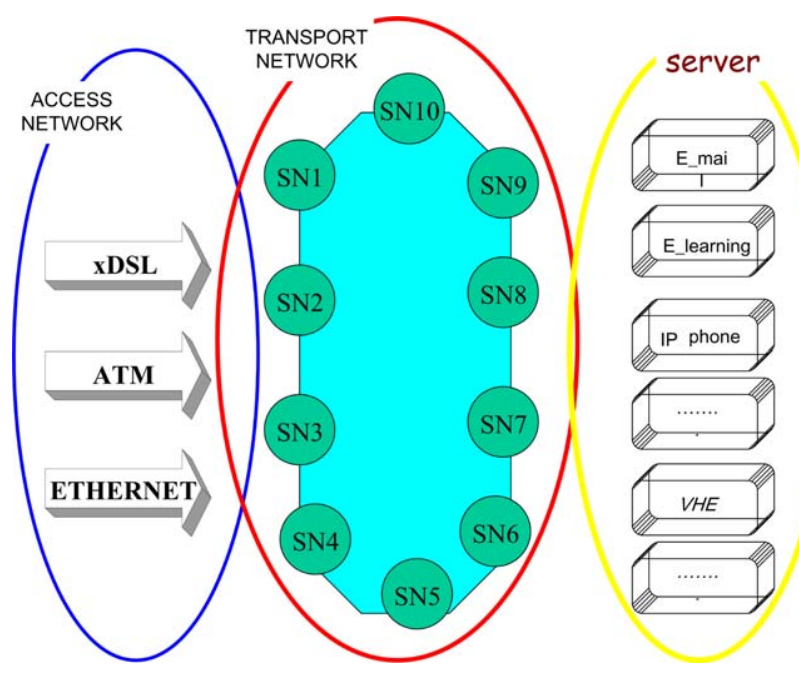

Fig. 5. Network of the University of Catania.

just the telephone exchange, being independent of all the others and disconnected from the data network. However, the installation of these PBXes is previous to the implementation of the broadband metropolitan area network, recalled over.

In 2003, a process of widening and renewal of voice and data network began. The presence of a broadband network infrastructure initially pushed the design engineers to plan a change of data and voice systems towards a convergent solution.

The new installations were carried out in four university departments in which network infrastructure and systems for data transmission and telephone transmission were implemented ex-novo. Structured in-building wiring to the desktop, dedicated switched Ethernet to each telephony desktop and IP telephony powering were taken into account. The investment, in terms of telephone system, concerned: the set-up of approximately 1000 network sites resorting to the technique of structured cabling; switch supply for 800 customer accesses; the implementation of 3 star centers interconnected to the broadband metropolitan area network and the supply of approximately 520 IP phones. All systems were set-up using Cisco AVVID technology. Subsequently, in an experimental way, a VoIP system based on open source software was interconnected.

In planning such systems, three requirement typologies were taken into account:

- general requirements strictly related to the saving of the system management costs and of the telephone traffic costs among the university centers;

- specific requirements regarding the cost saving of maintenance and training;

- technical requirements regarding cost/performance ratios on switches, servers, and cabling.

With the above-mentioned requirements, a cost analysis was made. At the same time, the break-even-point was calculated. This was compared to the one that University could support if the traditional data and telephone systems were chosen.

The concerned costs were:

- cabling;

- switches, routers, voice gateways, and VoIP servers;

- deployment speed;

- management and administration of users and network; - training.

Moreover, the phone call saving among interconnected users by means of the data network and the cost of the leased lines necessary to interconnect the classical PBX were considered.

Table 1 shows in a synthetic way the calculated costs on a temporal basis of 3 years, to set up a system for data and voice transmission based on a convergent solution or on a traditional one. The costs calculated for the convergent solution refer to the market costs in case of a solution based on system Cisco/AVVID and are derived from some public tenders for contract and real prices that university suppliers make.

The costs, in Table 1, for the cabling implementation in the convergent case of solution are reduced because the connections between the nodes are exclusively realized by 
Table 1

Costs of the convergent solution compared to those of a traditional solution

\begin{tabular}{llll}
\hline Costs & Time & Convergent & Traditional \\
\hline Cabling & 0 & 130 & 170 \\
Data/voice devices & 0 & 420 & 250 \\
Deployment & 0 & 25 & 45 \\
Training & 0 & 10 & 30 \\
Management & 1 & $\approx 0$ & 6 \\
& 2 & $\approx 0$ & 6 \\
& 3 & $\approx 0$ & 6 \\
Maintenance & 1 & 38 & 57 \\
& 2 & 40 & 60 \\
Telephone traffic & 3 & 42 & 63 \\
& 1 & 18 & 45 \\
Interconnection & 2 & 18 & 45 \\
& 3 & 18 & 45 \\
& 1 & 0 & 15 \\
& 2 & 0 & 15 \\
& 3 & 0 & 15 \\
\hline
\end{tabular}

The values are in KEuro.

means of optic fibre links, and therefore the multibrace cables are not necessary.

The major direct measurable cost, in Table 1, is certainly the "cost for the material supply " necessary for the convergent voice/data system operation.

The installation cost are calculated by estimating the deployment speed, that is the time necessary to make the telephone user operative. It considers the time necessary to permute cables in case of traditional telephone systems or to carry out auto-discovery of the IP phones in case of a VoIP solution. The complete installation cost of the single telephone is calculated assuming that the total time necessary to install 5 telephones is $1 \mathrm{day} / \mathrm{man}$ in a traditional system, while, at the same time, in a VoIP system, 20 IP telephones are settled. The configuration also includes initial telephone personalization and supply to each customer of the first information in order to use telephone.

Analyzing Table 1 is obviously the remarkable difference between the set-up cost of the convergent system and the one of the traditional telephony system, because the traditional telephony installation involves more time for the final realization because of the lack of flexibility and scalability typical, instead, of a convergent system.

The device management costs, in case of a convergent solution, are assumed to be nearly to zero. This results from the fact that the university technical staff are appointed to manage the data transmission devices, and the work increase due to the specific telephone equipment in the convergent solution turns out to be considerable. In the cost evaluation, the operations of moving, adding, and changing telephone extensions are considered. The unit cost of such activity are calculated by analyzing the costs supported over years in other university centers. Generally, the cost is nearly 15,000 euro for a single intervention made by personnel being not employed by the University, and it approximately concerns $10 \%$ of the customers during the whole year. The number of interventions varies according to the typology of university departments: it is usually limited in case of ordinary departments, it turns out to be more relevant in case of scientific departments that are characterized by an increasing staff mobility.

The maintenance costs in case of a traditional system are, in general, higher than those of a convergent system because the same IP network is used for both data and voice transmission. Therefore, in case of a traditional telephony system the annual licence fee could be higher because this is divided into two parts:

- one destined to the maintenance data network;

- the other to the maintenance voice network.

For it regards the training costs, the obvious saving is gained by a smaller number of days/man necessary to teach the system running. Presently, a big amount of the traditional system is based on an obsolete operation logics, often complex and therefore difficult to be understood if compared to the extremely easy use of a system based on IP, whose administration does not demand any specific technical knowledge.

Moreover, in the specific case of the University of Catania, the cost difference for maintenance and formation activities between the traditional solution and the convergent one is still higher. Actually, the University of Catania organized an internal technical staff for data network, while it must always rely on external staff as far as the traditional telephone systems are concerned.

A further advantage that results from the use of an integrated solution voice/data is also the possibility of considerably decreasing costs of telephone traffic, using the technique of the "toll bypass". This technique takes advantage both of the university centers, spread on the regional territory and the customers who find themselves in a foreign country in some periods of the year. On the contrary, this is not used for communications in a urban area, because of the low cost of calls.

The "toll bypass" can be associated to another technique", the "long reach by pass". This one takes advantage of a geographical data network also for calls to customers outside scattered university sites. Such method, together with toll bypass, determines a real saving of telephone traffic cost equal to $60 \%$.

Finally, the interconnection cost, in the specific case of the University of Catania, turns out to be zero for data transmission, and therefore for the convergent solution, because the University has a network distributed in the metropolitan area. The comparison with a traditional system was carried out if leased E1 lines of $2.048 \mathrm{Mbit} / \mathrm{s}$ are used.

In the end, the break-even-point analysis shows, how at " 0 " time, the University made a greater investment for the acquisition of the convergent solution rather than the tra- 
ditional one, while for the subsequent years (time 1,2,3) a clean cost saving was verified. The calculations, brought back in table, are obviously indicative and they refer to a specific case implemented at the University of Catania. Also in the case of traditional solutions they can use techniques reducing some cost items (for example, the interconnection cost can be reduced by using tributary interface on ATM SDH installed in the main nodes of the University network), even if they can consequently increase other cost items (for example, equipment cost). However, in any case, this analysis puts in evidence a reduction of all the supported costs over time.

At the end of the analysis, the savings on the operational costs were estimated: this pushed to choose a convergent solution.

The cost forecast was shared with the technicians of the University. Later on, for the system activation, the customers and the technicians of the University were asked to express a judgement about the system quality, that is, to express a judgement on the Assessed QoS. The analysis result was that:

- the customers with poor familiarity with the computer technologies initially expressed a bad opinion, because they considered the use of the IP phone as a complex one, as well as the voice signal quality, if compared to that of the traditional PBX;

- after a short period of VoIP service use (i.e., voice, mail, news, etc.) the judgement became positive;

- the customers accustomed to the computer technologies expressed a positive judgement;

- the technicians expressed an excellent judgement because, they had an appropriated know-how and appropriated expertise, they were able to appreciate the effectiveness of a voice-data integrated solution.

\section{Related work}

Technological improvement, savings of money, and customer satisfaction are three important variables that are taking into account to evaluate any emerging solution and so a VoIP one.These concepts have been widely discussed in literature and there are different kinds of analysis driven sometimes from one variable and sometimes from another one. For example, Heron et al. [18] analyze VoIP from only a technology point of view. Actually, exploring the capabilities a particular product offers and predicting what it may be possible to deliver in the future falls within the realm of technology evaluation and assessment. Heron et al. start their analysis considering the major stakeholders in the VoIP industry and their relative point of view because technology evaluation is much broader than the simple testing of products and solutions against a test specification.It is important to highlight functional and no-functional characteristics.
Reynolds et al. [19] evaluate VoIP quality concentrating on speech quality and end-to-end delay because they regard that the future success of VoIP will be strongly influenced by customer opinions of call quality (intrinsic quality of service) and how this quality compares with that of the public switched telephone network (PSTN). In our work, we want to give a full judgment about VoIP, in a word we evaluate the assessed quality of service. We consider more variables (judgment of various customer, money saving in the long time, and so on) and propose a economical methodical evaluation of a VoIP in comparison with PSTN.

\section{Conclusions}

In this article we mean to offer a brief assessment of the VoIP systems. We perform an analysis from both a merely technological point of view and an economical one. Our work has outlined the new aspects and the advantages of a convergent solution. The analysis has been led in a pragmatic way by testing the system inside the University of Catania. The advantages in terms of management easiness, better performance, and lower costs are undeniable. The results of the analysis have been synthesized in a high value of assessed quality of service. This has resulted from the heterogeneity of the actors in the above-mentioned environment. The choice of the VoIP technology is winning because it allows to integrate all IT components into one system only. Now, we try to evaluate security problem in a VoIP system with an adequate risk analysis. For this work we adopt a risk analysis methodology that we implemented and showed in an international conference. It is an ad hoc method for risk assessment of distributed component as VoIP is.

\section{References}

[1] B. Goode, Voice over internet protocol (VoIP), Proc. IEEE 90 (9) (2002) 1495-1517.

[2] M. Gaynor, Linking market uncertainty to VoIP service architectures, IEEE Internet Comput. (2003) 16-22.

[3] D.A. Das, Convergence in technology and regulation. The IP Telephone Case, in: Proc. IEEE ICMIT 2000, (2000), pp. 168-173.

[4] S. Taylor, VoIP state of the market report, Webtorials (2003).

[5] V. Firoiu, J. Le Boudec, D. Towsley, Z.L. Zhang, Theories and models for internet quality of service, Proc. IEEE 90 (9) (2002) 1565 1591.

[6] W.C. Hardy, QoS Measurement and Evaluation of Telecommunications Quality of Service, Wiley, New York, 2001.

[7] J. Gozdecki, A. Jajszczyk, R. Stankiewicz, Quality of service terminology in IP networks, IEEE Commun. Mag. 41 (3) (2003) 153-159.

[8] E. Crawley et al., A Framework for QoS-Based Routing in the Internet, IETF RFC 2386, (1998).

[9] R. Braden, D. Clark, and S. Shenker, Integrated Services in the Internet Architecture: An Overview, IETF RFC 1633, (1993).

[10] S. Blake et al., An architecture for differentiated services, IETF RFC 2475, (1998).

[11] ETSI. Network Aspects (NA): General Aspects of Quality of Service (QoS) and Network Performance (NP). Technical Report ETR003, 2nd ed., (1994). 
[12] ITU-T. Terms and definitions related to Quality of Service and Network Performance including Dependability. ITU-T Rec. E.800, (1993).

[13] ITU-T. General Aspects of Quality of Service and Network Performance in Digital Networks, including ISDN, ITU-T Rec. E.350, (1993).

[14] ITU-T. Internet Protocol Data Communication Service-IP Packet Transfer and Availability Performance Parameters. ITU-T Rec. Y.1541, (1999).

[15] A. La Corte, A. Lombardo, S. Palazzo, G. Schembra, Control of perceived quality of service in multimedia retrieval services: prediction-based mechanism vs. compensation buffers, Multimedia Syst. 6 (2) (1998) 102-112.

[16] W. Radinger and M. Goeschka, A definition of convergence in the area of information and telecommunication technologies. Conference on Object Oriented Programming Systems Languages and Applications archive Companion of the 17th annual ACM SIGPLAN conference on Object-oriented programming, systems, languages, and applications, (2002) 88-89.

[17] R. Steinmetz, K. Nahrstedt, Multimedia — Computing, Communications, and Applications, Prentice Hall International, New York, 1995.

[18] A.J. Heron, A.H. Warren, Evaluating VoIP technology-technology push/market pull, BT Technol. J. 19 (2) (2001) 158-167.

[19] R.J.B. Reynolds, A.W. Rix, Quality VoIP-an engineering challenge, BT Technol. J. 19 (2) (2001) 23-32.

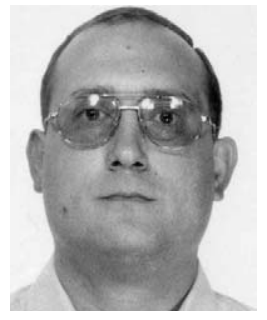

Aurelio La Corte received the degree in electrical engineering from University of Catania and the $\mathrm{Ph} . \mathrm{D}$. degree in electronic and computer science in 1988 and 1992, respectively. Since 1994 he has been at the University of Catania, where is now an Associate Professor of Telecommunications Networks. His current research interests include mobile systems, quality of service management, multimedia traffic model, and digital signal processing. His mail address is aurelio.lacorte@ unict.it.

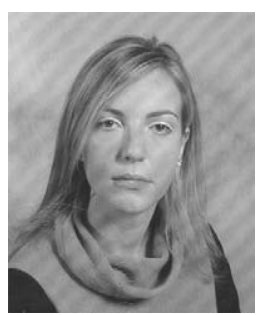

Sabrina Sicari received her degree in Electronic Engineering from University of Catania, Italy in 2002. Since 2003 she has been at the University of Catania, where is currently a Ph.D. student with the Department of Computer Science and Telecommunication Engineering. Since 2004 she collaborates for some researches with DEI, Politecnico of Milan. Her current research interest includes quality of service, mobile communication, network security, and risk analysis. Her mail address is sabrina.sicari@unict.it. 